\title{
Remoção de introdutor arterial pós-intervenção coronária percutânea: médico residente versus enfermeiro especializado
}

\author{
Arterial sheath removal after percutaneous coronary intervention: \\ resident versus specialized nurse
}

\begin{abstract}
José D el C armen Solano', G eorge C esar Ximenes M eireles², Luciano M auricio de Abreu³, Antonio Artur da C ruz Forte', M arcos Kiyoshi Sumita ${ }^{3}$, Jorge $\mathrm{H}$ ideki $\mathrm{H}_{\text {ayashi }}{ }^{3}$
\end{abstract}

\section{Resumo}

O bjetivo: Comparar os resultados da retirada de introdutor arterial pelo enfermeiro especializado em Unidade de Hemodinâmica e pelo médico residente em Cardiologia Intervencionista em pacientes submetidos à intervenção coronária percutânea.

M étodos: Trata-se de registro prospectivo em 100 pacientes submetidos à intervenção coronária percutânea, no período de setembro a outubro de 2004, divididos em dois grupos: G rupo A (GA) - enfermeiro ( $n=48$ pacientes) - eG rupo $B(G B)$ - médico residente $(n=52$ pacientes). $\mathrm{H}$ ematoma pequeno foi definido como inchaço palpável no local da punção menor que $2 \mathrm{~cm}$; hematoma moderado, com 2 a 6 $\mathrm{cm}$ de diâmetro; e hematoma grande, maior que $6 \mathrm{~cm}$ de diâmetro. A dose de heparina foi de $100 \mathrm{UI} / \mathrm{kg}$. O s introdutores foram retirados após controle do tempo de coagulação ativado (TCA < 180 segundos), e foi realizada compressão manual por 15 minutos.

Resultados: A idade dos pacientes foi de $59,54 \pm 11,1$ anos (GA) e $61,7 \pm 10,4$ anos $(G B)$, com predomínio do sexo masculino ( $\mathrm{GA}=75 \%$ e $\mathrm{GB}=58 \%$ ). O s introdutores foram 7 French. 0 tempo de compressão manual foi de 19,4 $\pm 3,1$ minutos no $G A$ e 19,6 $\pm 3,1$ minutos no $G B(P=0,76)$. 0 correram oito hematomas no $G A$ (sete pequenos e um moderado) e nove hematomas no $G B$ (sete pequenos e dois moderados), $P=$ não-significante. $O$ s hematomas foram tratados clinicamente, sem complicações.

Conclusão: A retirada de introdutor arterial, após intervenções coronárias percutâneas, pode ser realizada pel o enfermeiro especializado em Unidade de $\mathrm{H}$ emodinâmica ou pel o médico residente em Cardiologia Intervencionista com segurança e sem complicações maiores.

Palavras-chave: Angioplastia transluminal percutânea coronária, enfermagem, capacitação.

\begin{abstract}
O bjective: To compare the results of sheath removal by the catheterization lab specialist nurse and by the interventional cardiology resident in patients submitted to a percutaneous coronary intervention.

Methods: Prospective study with 100 patients submitted to percutaneous coronary intervention, from September to 0 ctober 2004, who were divided into two groups: Group A (GA) - nurse $(n=48)$ and Group $B(G B)$ - resident $(n=52)$. Small hematoma was defined as a palpable swelling at the access site measuring less than $2 \mathrm{~cm}$; mild hematoma, from 2 to $6 \mathrm{~cm}$ in diameter; and large hematoma when it was larger than $6 \mathrm{~cm}$ in diameter. The heparin dose was $100 \mathrm{IU} / \mathrm{kg}$. The sheaths were removed after activated coagulation time control (ACT < 180 seconds) and a 15-minute manual compression was used.
\end{abstract}

Results: Patients' age was $59.54 \pm 11.1$ (GA) and $61.7 \pm 10.4$ $(G B)$ years with a predominance of male patients ( $G A=75 \%$ and $\mathrm{GB}=58 \%)$. $7 \mathrm{~F}$ sheaths were used. $\mathrm{M}$ anual compression time was $19.4 \pm 3.1 \mathrm{~min}$ for $\mathrm{GA}$ and $19.6 \pm 3.1 \mathrm{~min}$ for $\mathrm{GB}(P=0.76)$. There were eight hematomas in $\mathrm{GA}$ (seven small and one mild) and nine hematomas in GB (seven small and two mild), P = nonsignificant. The hematomas were clinically treated, with no complications.

Conclusion: Arterial sheath removal, after percutaneous coronary interventions, can be made by the catheterization lab specialist nurse or interventional cardiology resident safely and without major complications.

Key words: Percutaneous transluminal coronary angioplasty, nursing, training.

1. Médico residente do Serviço de Hemodinâmica e Cardiologia Intervencionista, Hospital Stella Maris, Guarulhos, SP.

2. Doutor em Cardiologia, Instituto do Coração, Faculdade de Medicina da Universidade de São Paulo (FMUSP), São Paulo, SP.

3. Médicos assistentes, Serviço de Hemodinâmica e Cardiologia Intervencionista, Hospital Stella Maris, Guarulhos, SP.

Este estudo foi apresentado no XXVII Congresso da Sociedade Brasileira de Hemodinâmica e Cardiologia Intervencionista e no XI Congresso da Sociedad Latinoamericana de Cardiologia Intervencionista.

Artigo submetido em 25.11.05, aceito em 20.03.06. 
0 manuseio do local da punção e a retirada do introdutor arterial após as intervenções coronárias percutâneas têm aspecto importante, pois estão relacionados às complicações hemorrágicas e vasculares, ocasionando aumento da morbidade e dos custos hospitalares.

Com a utilização do corrente regime antiplaquetário de AAS, associado à ticlopidina ou clopidogrel pósimplante destent, podem ocorrer complicações hemorrágicas locaismaiores em, aproximadamente, 2,5\% dos pacientes $^{1,2}$. Foi estimado queas complicaçõesmaiores do local de acesso arterial aumentaram a duração da internação hospitalar por aproximadamente 2 dias, adicionando U S\$2.000,00 ao custo total do procedimento $0^{3,4}$.

É prática comum no nosso país a retirada de introdutores arteriais e venosos pelos médicos residentes e, em al gumas instituições, por enfermeiro especializado em U nidade de $\mathrm{H}$ emodinâmica, mas ainda não foram realizados levantamentos das instituições que os realizam, nem estudos sobre os resultados destes procedimentos.

Para investigar a segurança da retirada de introdutor arterial após intervenções coronárias percutâneas pelo médico residente ou enfermeiro, foi elaborado 0 estudo RIPRE (R emoção de Introdutor Arterial Pós-I ntervenções Coronárias Percutâneas: médico residente versus enfermeiro).

\section{M étodos}

Trata-se de registro prospectivo da retirada do introdutor arterial por enfermeiro especializado em U nidadedeH emodinâmica versusmédico residenteem C ardiologial ntervencionista, em pacientessubmetidos a intervenções coronárias percutâneas. O s pacientes foram incluídos no período de setembro a outubro de 2004. 0 presente estudo foi avaliado e aprovado pelo Comitêde Pesquisa eÉtica da nossa instituição (protocolo 06/04, aprovado em agosto de 2004).

O s critérios de inclusão foram: ser elegível para revascularização miocárdica percutânea ou cirúrgica; pacientes com diagnóstico deangina estável ou instável e infarto agudo do miocárdio. O s critérios de exclusão foram: complicações vasculares durante a intervenção coronária percutânea; presença de hematoma após a punção da artéria femoral; doença vascular arterial periférica; moléstias hemorrágicas (púrpuras, coagulopatias, etc.); uso contínuo de anticoagulantes; terapia com fibrinolítico nasúltimas 48 horas; obesidade mórbida (índice de massa corpórea maior que 40).

As complicações foram assim definidas: sangramento maior, como sangramento intracraniano, ou diminuição na concentração de hemoglobina maior ou igual a $5 \mathrm{~g} / \mathrm{dl}$, ou diminuição do hematócrito maior ou igual a 15\%; sangramento menor, como diminuição na concentração dehemoglobinamaior que $3 \mathrm{~g} / \mathrm{dl}$ emenor que $5 \mathrm{~g} / \mathrm{dl}$, ou diminuição do hematócrito maior que $10 \%$ e menor que $15 \% 5$; complicação vascular perifé rica, como isquemia do membro inferior relacionada à punção arterial, pseudo-aneurisma, fístula arteriovenosa ou operação para reparo de lesão vascular ${ }^{5,6}$; hematoma pequeno, com inchaço no local da punção menor que $2 \mathrm{~cm}$, hematoma moderado, com 2 a $6 \mathrm{~cm}$ de diâmetro, e hematoma grande, maior que $6 \mathrm{~cm}$ de diâmetro ${ }^{7}$.

Foram utilizadoso introduvor 7 F rench eo cateterguia de diâmetro igual a 7 French pela via femoral.

O s pacientes foram medicados com aspirina 200 $\mathrm{mg}$ ao dia e clopidogrel $300 \mathrm{mg}$ no dia anterior ao procedimento. A heparinanadosede $100 \mathrm{Ul} / \mathrm{kg}$ por via endovenosa foi aplicada antes do implante. A pós a alta hospitalar, o clopidogrel $75 \mathrm{mg}$ foi administrado por 30 dias e a aspirina indefinidamente.

O s introdutores foram retirados após controle do tempo de coagulação ativado (menor que 180 segundos), seguido por compressão manual por período mínimo de 15 minutos. Após este período, foi avaliada a presença de sangramento local e, se necessário, prolongada a compressão, com avaliaçõesa cada 5 minutos, até a completa hemostasia.

Após o procedimento de retirada do introdutor do acesso arterial, foi verificada, por um médico assistente do serviço, a presença de hematoma e/ ou sangramento local, e foram avaliados os pulsos do membro inferior no qual foi o retirado o introdutor, assim como a perfusão e diferenças na temperatura. Os pacientes permaneceram deitados no leito por um período mínimo de 6 horas, com o membro em repouso horizontal.

Foram obtidosum hemograma eum coagulograma antes do implante destent eum hemograma etempo de coagulação ativado 4 horas após o procedimento.

C aso não houvesse qualquer complicação, programou-se a alta hospitalar para 24 a 48 horas após 0 implante de stent.

As variáveisquantitativasforam apresentadascomo médias e desvios padrão. Foram cal culadas as freqüên- 
cias absolutas e relativas às variáveis qualitativas. Para a análise estatística das variáveis contínuas, foi usado o teste t de Student, e para comparação das freqüências, 0 teste exato de Fisher.

O sdadosforam colhidos em formulário individual para cada paciente, com as informações sobre os dados demográficos, os fatores de risco e os relacionados ao procedimento.

\section{Resultados}

No período de setembro a outubro de 2004, foram incluídos 100 pacientes consecutivos, divididos em dois grupos: Grupo $A(G A)$ - enfermeiro ( $n=48$ pacientes) - e Grupo $B(G B)$ - médico residente( $n=52$ pacientes). Foi excluído um paciente devido à presença de hematoma após a punção. $A$ idade dos pacientes foi de 59,54 $\pm 11,1$ anos (G A) e $61,7 \pm 10,4$ anos (GB), com predomínio do sexo masculino ( $G A=75 \%$ e GB $=58 \%$ ). Ascaracterísticas basais dos pacientes não apresentaram diferença estatisticamente significante (T abela 1).

T odos os pacientes fizeram uso de $200 \mathrm{mg}$ de AAS e300 mg declopidogrel no dia anterior ao procedimento. As vias de acesso foram as artérias femorais direita
( $G A=68,75 \%$ eG $B=76,9 \%$ ) eesquerda( $G A=31,25 \%$ e $G B=23,1 \%$ ). 0 s introdutores foram 7 French. 0 implante de stent coronário foi realizado em $100 \%$ dos pacientes do GA e $98,1 \%$ dos pacientes do $G B$, e 0 sucesso do procedimento ocorreu em $100 \%$ das intervenções no $\mathrm{GA}$ e em $98,1 \%$ no $\mathrm{GB}$.

Em relação ao tempo de retirada dos introdutores, tempo de compressão e formação de hematoma, não ocorreram diferenças estatisticamente significantes entre os dois grupos. Os hematomas foram tratados clinicamente, evoluindo sem complicações (T abela 2).

\section{D iscussão}

Este é o primeiro estudo que compara a segurança da retirada de introdutor arterial pel o enfermeiro especializado em U nidade de $\mathrm{H}$ emodinâmica ou pelo mé dico residente em $\mathrm{C}$ ardiologia Intervencionista, após intervençõescoronárias percutâneasna prática diária de um Serviço de Cardiologia Intervencionista.

N o Brasil, a retirada do introdutor arterial após procedimentos coronários percutâneos diagnósticos e terapêuticos pode ser realizada por profissional enfermeiro, desde que o mesmo tenha se submetido a um curso de especialização em Enfermagem em T erapia

Tabela 1 - Características basais dos 100 pacientes

\begin{tabular}{lcc}
\hline Características basais & $\begin{array}{c}\text { GA (\%) } \\
\text { (n = 48) }\end{array}$ & $\begin{array}{c}\text { G B (\%) } \\
\text { (n = 52) }\end{array}$ \\
\hline Sexo (masculino/feminino) & $75 / 25$ & $58 / 42$ \\
Idade (anos) & $59,54 \pm 11,1$ & $61,7 \pm 10,4$ \\
H ábito de fumar atual/passado* & $4,2 / 52,1$ & $7,7 / 51,9$ \\
Antecedente familiar para doença coronária & 2 & 7,7 \\
Intervenção coronária percutânea prévia & 30,8 & 31,25 \\
Punção femoral prévia & 39,6 & 32,73 \\
H ipertensão arterial sistêmica ( $\geq 140 / 90$ mmH g) & 90,4 & 85,4 \\
D iabetes melito ( $\geq 126$ mg/dl) & 25 & 40,4 \\
H ipercolesterolemia ( $\geq 200$ mg/dl) & 70,2 & 67,3 \\
Infarto do miocárdio prévio (>180 dias) & 20,8 & 28,9 \\
Intervenção coronária percutânea prévia & 31,2 & 30,8 \\
Síndrome coronariana aguda & 45,8 & 32,7 \\
Angina estável & 54,2 & 67,3
\end{tabular}

* Passado = abandono há mais de um mês e inferior a um ano.

$\mathrm{GA}$ = grupo atendido pela enfermagem; $\mathrm{GB}$ = grupo atendido pelo médico residente

$P=$ não-significante para todas as variáveis. 
T abela 2 - Resultados

\begin{tabular}{lccc}
\hline Variáveis & $\begin{array}{c}\text { GA } \\
\text { (n= 48) }\end{array}$ & $\begin{array}{c}\text { G B } \\
(\mathbf{n}=\mathbf{5 2})\end{array}$ & P \\
\hline Tempo de retirada do introdutor $(\mathrm{min})$ & $268,2 \pm 28,4$ & $269,54 \pm 54,4$ & 0,87 \\
Tempo de compressão manual $(\mathrm{min})$ & $19,4 \pm 3,1$ & $19,6 \pm 3,3$ & 0,75 \\
Formação de hematoma & 8 & 9 & \\
H ematoma pequeno & 7 & 7 & \\
H ematoma moderado & 1 & 2 & 1,0 \\
\hline
\end{tabular}

$\mathrm{GA}=$ grupo atendido pela enfermagem; $\mathrm{GB}=$ grupo atendido pelo médico residente.

Intensiva ou Enfermagem em U nidade de H emodinâmica, poiso procedimento écomplexo epodeacarretar sérios riscos ao paciente ${ }^{8}$.

A vantagem da capacitação do enfermeiro para a retirada do introdutor arterial éque um maior número de profissionais que trabal ham na equipe de C ardiologial ntervencionista estarácapacitado para esteprocedimento, com diminuição da sobrecarga de trabalho entre os seus membros.

É crescente a prática da retirada de introdutores arteriais e venosos pelo enfermeiro após intervenções coronárias percutâneas no Canadá, EUA e Inglaterra9-13. N o Canadá, essa participação ocorre em aproximadamente um quarto das instituições ${ }^{10}$, e o grau de satisfação dos enfermeiros com esta mudança na prática foi obtida em $46 \%$ dos entrevistados ${ }^{14}$.

Juran et al. ${ }^{9}$, em estudo prospectivo multicêntrico com 4.000 pacientes submetidos a intervenções coronárias percutâneas, mostraram significante correlação entre as intervenções pelo enfermeiro ea ocorrência de sangramento de moderado a intenso no local do acesso arterial, sem maiores repercussões. Os fatores mais significantes na diminuição de complicação no local do acesso arterial foram o menor tempo decorrido para a remoção do introdutor arterial, o tipo de mecanismo usado para a obtenção da hemostasia, a alocação profissional e o método utilizado na remoção.

Q uando comparadas as técnicas de compressão manual emecânica para a retirada do introdutor arterial femoral realizadas pelo enfermeiro, não se observa diferença em relação aos sangramentos; contudo, há aumento de hematomas no grupo com compressão manual ${ }^{15}$.

Em outubro de 2001, foi implementado um programa para a capacitação da retirada precoce do introdutor arterial pós-intervenções coronárias percutâneas pel os enfermeiros da U nidade de C ardiologia do M assachusetts $\mathrm{G}$ eneral Hospital, e observou-se que 106 introdutores foram removidos durante o mês de novembro de 2001 , e, destes, $58 \%$ o foram pelosenfermeiros, sendo observada a formação dehematoma em nove pacientes $(8,5 \%)$, taxa comparável quando realizados pelos médicos ${ }^{16}$.

Em conclusão, a retirada deintrodutor arterial pelo enfermeiro especializado em U nidade de $\mathrm{H}$ emodinâmica ou pelo médico residente em $\mathrm{C}$ ardiologia Intervencionista mostrou-se um procedimento seguro, sem aumento de complicações. É importante salientar a importância do treinamento especializado para esses profissionais para a obtenção de bons resultados.

\section{Referências}

1. Bertrand ME, Legrand V, Boland J, et al. Randomized multicenter comparison of conventional anticoagulation versus antiplatelet therapy in unplanned and electivecoronarystenting - The Full Anticoagulation Versus Aspirin and Ticlopidine (FAN TASTIC) study. Circulation. 1998;98:1597-603.

2. Leon M B, Baim DS, Popma J), et al. A clinical trial comparing three antithrombotic-drug regimens after coronary artery stenting. N Engl J Med. 1998;339:1665-71. 
3. Dick RJ, Popma JJ, M uller DW, Burek KA, Topol EJ. Inhospital costs associated with new percutaneous coronary devices. Am J Cardiol. 1991;68:879-85.

4. K rause PB, Klein LW. U tility of a percutaneous collagen hemostasis device: to plug or not to plug? J Am Coll Cardiol. 1993;22:1280-2.

5. Cote AV, Berger PB, Holmes DR, Scott CG, Bell MR. $\mathrm{H}$ emorrhagic and vascular complications after percutaneous coronary intervention with adjunctive abciximab. $\mathrm{M}$ ayo $\mathrm{Clin}$ Proc. 2001;76:890-6.

6. Smith SC Jr, D oveJT, JacobsAK, et al. ACC/AH A guidelines of percutaneous coronary interventions (revision of the 1993 PTCA guidelines): executive summary. J Am Coll Cardiol. 2001;37:2215-39.

7. Schickel SI, Adkisson P, M iracle V, Cronin SN . Achieving femoral artery hemostasis after cardiac catheterization: a comparison of methods. Am J Crit Care. 1999;8:406-9.

8. Conselho Regional de Enfermagem. Parecer técnico do Conselho Regional de Enfermagem (COREN) do Distrito Federal no 014/2001 - Competência legal do profissional enfermeiro na retiradadecateter introdutor apósprocedimentos denatureza hemodinâmica[monografia nal nternet]. D istrito Federal: COREN ; 2001 [citado 2001 nov.]. D isponível em: http://www.coren-df.org.br.

9. Juran N B, Rouse CL, Smith D D, O 'Brien M A, D el uca AS, Sigmon K. N ursing interventions to decrease bleeding at the femoral access site after percutaneous coronary intervention. SAN D BAG N ursing C oordinators. Standards of angioplasty nursing techniques to diminish bleeding around the groin. Am J C rit Care. 2000;8:303-13.

10. Peet GI, M CG rath M A, Brunt JH , H ilton JD. Femoral arterial sheath removal after PTCA: a cross-Canada survey. Can J Cardiovasc N urs. 1995;6:13-9.

11. Schickel S, Cronin SN, M ize A, Voelker C. Removal of femoral sheaths by registered nurses: issues and outcomes. Crit Care N urse. 1996;16:32-6.
12. O 'G rady $E$. Removal of a femoral sheath following PT CA in cardiac patients. Prof N urse. 2002;17:651-4.

13. Smith TT, Labriolola R. D eveloping best practice in arterial sheath removal for registered nurses. J N urs Care Qual. 2001;16:61-7.

14. Rubins $S$, W iens $L$, Fingler I, Sawyer $T$, G arcia-Vargas $P$, Stovicek $Z$. Evaluating a change in practice: femoral sheath removal by registered nurses. Can J Cardiovasc $\mathrm{N}$ urs. 1996;7:19-27.

15. Jones $T, M C C$ utcheon $H$. A randomised controlled trial comparing the use of manual versus mechanical compression to obtain haemostasis following coronary angiography. Intensive C rit Care N urs. 2003;19:11-20.

16. H aldemen S, Silva J, Gonzalez C, Carroll DL. Registered nurse sheath removal team: a quality improvement initiative. In: M assachusetts General H ospital, N ursing Research Committee. 2002 abstracts. Boston: M G H ; 2002 [citado 2002 dez.]. D isponível em: http://www.massgeneral.org/pcs/ ccpd/N ursing_Research/Research_Abstracts_2002.asp.

\section{Correspondência:}

G eorge C esar Ximenes M eireles

H ospital Stella M aris, Guarulhos, SP.

Rua M aria Cândida Pereira, 770, Itabegica

CEP 07041-020 - Guarulhos, SP

Tel.: (11) 6421.2833

Fax: (11) 6424.3218

E-mail: george@tre-sp.gov.br 